BMJ Open

Diabetes

Research

\& Care

\title{
Role of fructosamine-3-kinase in protecting against the onset of microvascular and macrovascular complications in patients with T2DM
}

\author{
Giovanni Sartore, ${ }^{1}$ Eugenio Ragazzi (D) , ${ }^{2}$ Silvia Burlina, ${ }^{1}$ Renata Paleari, ${ }^{3,4}$ \\ Nino Cristiano Chilelli, ${ }^{1}$ Andrea Mosca, ${ }^{3,4}$ Francesca Avemaria, ${ }^{3}$ \\ Annunziata Lapolla ${ }^{1}$
}

To cite: Sartore G, Ragazzi E, Burlina S, et al. Role of fructosamine-3-kinase in protecting against the onset of microvascular and macrovascular complications in patients with T2DM. BMJ Open Diab Res Care 2020;8:e001256. doi:10.1136/ bmjdrc-2020-001256

- Additional material is published online only. To view, please visit the journal online (http://dx.doi.org/10.1136/ bmjdrc-2020-001256).

Received 5 February 2020 Revised 2 April 2020 Accepted 30 April 2020

Check for updates

(C) Author(s) (or their employer(s)) 2020. Re-use permitted under CC BY-NC. No commercial re-use. See rights and permissions. Published by BMJ.

For numbered affiliations see end of article.

Correspondence to Dr Eugenio Ragazzi; eugenio.ragazzi@unipd.it

\section{ABSTRACT}

Introduction Microangiopathic and macroangiopathic complications are the main cause of morbidity and mortality in the diabetic population. Numerous publications have highlighted the role of glycation in the onset of complications of diabetes. In this context, the detection of fructosamine3-kinase (FN3K) - an enzyme capable of counteracting the effect of hyperglycemia by intervening in protein glycationhas attracted great interest. Several studies have linked FN3K genetic variability to its enzymatic activity and glycated hemoglobin $(\mathrm{HbA1c})$ levels. Here, we investigated the role of FN3K polymorphisms in the development of microvascular and macrovascular complications of diabetes.

Research design and methods The anthropometric and biochemical parameters, and any medical history of microangiopathic and macroangiopathic complications, were documented in a sample of 80 subjects with type 2 diabetes. All subjects were screened for FN3K gene and analyzed for the combination of three polymorphisms known to be associated with its enzymatic activity (rs3859206 and rs2256339 in the promoter region and rs1056534 in exon 6). Results The combination of allelic variants of FN3K polymorphisms resulted in 13 distinct genotypic variants within the cohort. Comparison between genotypes showed no significant differences in terms of demographic, anthropometric and biochemical parameters, risk markers and long-term complications, except for a higher age and vitamin $E$ levels associated with the genotype presenting GG at position $-385, \pi$ at position -232, and CC at c.900 A. Evaluating the microangiopathic and macroangiopathic complications as a whole, we found that they appeared significantly less present in this genotype compared with all other genotypes $(p=0.0306)$.

Conclusions The group of patients carrying the favorable allele for the three polymorphisms of the FN3K gene revealed less severe microangiopathy and macroangiopathy, suggesting a protective role of this genotype against the onset of the complications of diabetes.

\section{INTRODUCTION}

Diabetes mellitus is a metabolic disorder that has become a public health problem because of its pandemic expansion worldwide. ${ }^{1}$ The global prevalence of diabetes in 2019 was estimated at $9.3 \%$ (amounting to 463 million

\section{Significance of this study}

What is already known about this subject?

- Fructosamine-3-kinase (FN3K) is an intracellular enzyme involved in deglycation, active in removing ketoamines and preventing advanced glycation end product production.

- Some polymorphisms of the FN3K gene are associated with variations in $\mathrm{HbA1c}$ levels and with the onset of type 2 diabetes mellitus (T2DM) and pathogenic mechanisms related to its complications.

What are the new findings?

- Allelic variants of FN3K polymorphisms revealed 13 distinct genotypic variants in a cohort of patients with T2DM.

- Comparison between genotypes indicated similar demographic, anthropometric and biochemical parameters, risk markers and long-term complications, except for a higher age and vitamin E levels associated with the genotype presenting $\mathrm{GG}$ at position $-385, \mathrm{TT}$ at position -232 , and CC at C. $900 \mathrm{~A}$.

- The FN3K genotype presenting GG at position -385, $\pi T$ at position -232 , and $\mathrm{CC}$ at c. $900 \mathrm{~A}$, is associated with less severe microangiopathic and macroangiopathic complications as a whole, compared to all other genotypes.

How might these results change the focus of research or clinical practice?

- The results suggest the opportunity of focusing research on FN3K genetic variability and on its potential applicability to the prevention, diagnosis and treatment of diabetes and its complications.

people), and it is expected to rise to $10.2 \%$ (578 million people) by $2030 .^{2}$ Diabetes is a complex heterogeneous disease in which genetic and environmental factors interact, causing hyperglycemia. Affected individuals are at high risk of morbidity and mortality due to the onset of microvascular and macrovascular complications. ${ }^{3}$ Many hypotheses have 
been advanced to explain the link between high blood sugar levels and the development of related complications. ${ }^{4-6}$ Non-enzymatic protein glycation is etiologically linked to diabetic complications ${ }^{6}$ and has been proven relevant in the diagnosis and treatment of diabetes, and the monitoring of a treatment's efficacy using glycated proteins such as $\mathrm{HbAlc}^{7}$ or fructosamine. ${ }^{8}$ Protein glycation is a common and spontaneous reaction dictated by blood glucose concentrations. It involves the binding of glucose or other reducing sugars to proteins, which leads to the formation of a broad and heterogeneous group of complex compounds called advanced glycation end products (AGEs). ${ }^{9}$ While the generation of Schiff base and Amadori-rearranged ketoamine products is reversible in normal reaction kinetics, ${ }^{10}$ there is another pathway for removing ketoamines and preventing AGE production catalyzed by fructosamine-3-kinase (FN3K). ${ }^{11} 12$ FN3K is an intracellular enzyme found expressed in tissues most prone to glycation, such as the heart, nerves and kidneys. ${ }^{13}$ Definitive evidence of FN3K involvement in deglycation has come from animal models, in which FN3K-knockout mice showed an increased protein glycation. ${ }^{14}$ In diabetic subjects, single-nucleotide polymorphisms were found to alter FN3K activity, affecting HbAlc levels, the onset of type 2 diabetes mellitus (T2DM), and pathogenic mechanisms related to its complications. ${ }^{13}{ }^{15}$ Hence, there is great interest in the identification of an enzyme, FN3K, as part of a protein repair system for opposing the consequences of hyperglycemia.

Table 1 Anthropometric, clinical and biochemical characteristics of the 80 patients with T2DM

\begin{tabular}{ll}
\hline Parameters (unit of measure) & \\
\hline Age (years) & $69 \pm 8$ \\
\hline Gender, M/F & $41 / 39(51 \%)$ \\
\hline Waist circumference (cm) & $102.4 \pm 10.9$ \\
\hline BMI (kg/m²) & $30.3 \pm 5.3$ \\
\hline Diastolic blood pressure (mm Hg) & $79 \pm 11$ \\
\hline Systolic blood pressure (mm Hg) & $139 \pm 14$ \\
\hline HbA1c (mmol/mol) & $54.8 \pm 12.8$ \\
\hline HbA1c (\%) & $7.2 \pm 1.2$ \\
\hline Total cholesterol (mg/dL) & $159 \pm 47$ \\
\hline HDL cholesterol (mg/dL) & $46 \pm 6$ \\
\hline Triglycerides (mg/dL) & $147 \pm 106$ \\
\hline Microalbuminuria, yes/no & $24 / 56(30 \% / 70 \%)$ \\
\hline Retinopathy, yes/no & $22 / 58(27.5 \% / 72.5 \%)$ \\
\hline Cerebral macroangiopathy, yes/no & $50 / 30(62.5 \% / 37.5 \%)$ \\
\hline Peripheral artery disease, yes/no & $11 / 69(13.8 \% / 86.2 \%)$ \\
\hline Coronary heart disease, yes/no & $4 / 76(5 \% / 95 \%)$ \\
\hline
\end{tabular}

Continuous variables are expressed as mean \pm SD. For frequency data, absolute values are given, with percentages in parentheses. $\mathrm{BMI}$, body mass index; F, female; HDL, high-density lipoprotein; $\mathrm{M}$, male; T2DM, type 2 diabetes mellitus.
In this study, we investigated the relationship between polymorphisms of the $F N 3 \mathrm{~K}$ gene involved in the deglycation system ( FN3K rs3859206, rs2256339 and rs1056534), and the onset of microvascular and macrovascular complications in individuals with T2DM.

\section{RESEARCH DESIGN AND METHODS \\ Patients}

Eighty consecutive patients with T2DM attending the outpatient Diabetes Center of the Department of Medicine at the University of Padova (Italy) were recruited. The mean age was $69 \pm 8$ years (range $48-85$ years, median 69 years), and the mean duration of diabetes was $14.6 \pm 4.3$ years (range $8-20$ years, median 15.5 years). T2DM was diagnosed in accordance with the WHO criteria ${ }^{1617}$ which require fasting plasma glucose higher than $126 \mathrm{mg} / \mathrm{dL}$. The sample's mean HbAlc levels were above the reference threshold (4\%-6\% in healthy people) ${ }^{18}$ and were associated with the presence of complications of diabetes. As inclusion criteria, we considered a stable glycemic control demonstrated by two HbAlc measurements in the 6 months prior to enrollment, with a variation not exceeding $1 \%$. Our exclusion criteria concerned all conditions that might cause changes in glycemia, such as disorders requiring steroid therapies or pregnancy, and any other conditions potentially affecting HbAlc concentrations (anemia, high erythrocyte turnover, bleeding, transfusions, hemoglobinopathies, liver and kidney diseases, and infections). The subjects included in the study were taking various treatments: 6 were on insulin therapy; 59 were on oral hypoglycemic therapy; and 15 were on both. During the clinical examination, the subjects' personal details (age and sex) and anthropometric data (BMI and waist circumference) were recorded, and their arterial blood pressure was measured. Peripheral venous blood samples were collected for biochemical and genetic analyses. For each subject, the following biochemical parameters were measured, using standard methods described elsewhere ${ }^{19}$ : HbAlc, total cholesterol, high-density lipoprotein cholesterol, triglycerides, homocysteine, vitamin $\mathrm{A}$ and vitamin $\mathrm{E}$.

Details of microangiopathic and macroangiopathic complications were obtained from the subjects' electronic medical records. Five parameters characterizing the microangiopathic and macroangiopathic complications typically associated with diabetes were examined: (1) microalbuminuria, calculated as the urine albumin:creatinine ratio (ACR), and classified as absent or present based on a cut-off of $30 \mathrm{mg} / \mathrm{g}$; (2) diabetic retinopathy, detected on examination of the fundus oculi and classified as absent or present; (3) cerebral macroangiopathy, investigated by echo color Doppler study of the supra-aortic vessels and classified as absent or present; (4) peripheral artery disease, detected by measuring the ankle/arm index (ABI index) and classified as absent or present based on a cut-off of 0.9 ; and (5) coronary heart disease, assessed on the basis of clinical documentation 
Table 2 Genotypes and allele frequencies of FN3K genetic variants identified in patients with T2DM

\begin{tabular}{|c|c|c|c|c|c|}
\hline Promoter region variants & & T2DM $(n=80)$ & Exon/intron variants & & T2DM $(n=80)$ \\
\hline \multirow[t]{4}{*}{ c. -385 A>G (rs3859206) } & AA & 0.31 & c. $2 \mathrm{~T}>\mathrm{A}$ & $\pi$ & 0.99 \\
\hline & $A G$ & 0.54 & & AT & 0.01 \\
\hline & GG & 0.15 & & AA & / \\
\hline & G-allele & 0.42 & & A-allele & 0.01 \\
\hline \multirow[t]{4}{*}{ c. -232 A >T (rs 2256339) } & AA & 0.27 & c.187 A>C (rs2253149) & AA & I \\
\hline & AT & 0.58 & & $A C$ & I \\
\hline & $\pi$ & 0.15 & & $\mathrm{CC}$ & 1 \\
\hline & T-allele & 0.44 & & C-allele & 0.01 \\
\hline \multirow[t]{4}{*}{ c. $-421 \mathrm{C}>\mathrm{T}$} & $\mathrm{CC}$ & 0.99 & IVS2-27 A>T & AA & 0.99 \\
\hline & $\mathrm{CT}$ & 0.01 & & AT & 0.01 \\
\hline & $\pi$ & / & & TT & I \\
\hline & T-allele & 0.01 & & T-allele & 0.01 \\
\hline \multirow[t]{24}{*}{ c.-429delATCGGAG } & +/+ & 0.99 & IVS2 +26 A>G (rs2253132) & AA & 1 \\
\hline & $+/$ del & 0.01 & & $A G$ & / \\
\hline & del/del & / & & GG & l \\
\hline & del & 0.01 & & A-allele & 0.01 \\
\hline & & & IVS2 +31 A>T (rs2253131) & AA & I \\
\hline & & & & AT & 0.19 \\
\hline & & & & $\pi$ & 0.81 \\
\hline & & & & A-allele & 0.09 \\
\hline & & & c. $465 \mathrm{G}>\mathrm{A}$ & GG & 0.99 \\
\hline & & & & $A G$ & 0.01 \\
\hline & & & & AA & l \\
\hline & & & & A-allele & 0.01 \\
\hline & & & IVS4-9delTTG (rs72318398) & +/+ & 0.74 \\
\hline & & & & $+/$ del & 0.25 \\
\hline & & & & del/del & 0.01 \\
\hline & & & & del & 0.14 \\
\hline & & & c. 900 C>G (rs1056534) & $\mathrm{CC}$ & 0.15 \\
\hline & & & & GC & 0.48 \\
\hline & & & & GG & 0.37 \\
\hline & & & & C-allele & 0.39 \\
\hline & & & c.906 C>T (rs149413139) & $\mathrm{CC}$ & 0.98 \\
\hline & & & & CT & 0.03 \\
\hline & & & & $\mathrm{TT}$ & / \\
\hline & & & & T-allele & 0.01 \\
\hline
\end{tabular}

Values for genotypes and rare alleles are frequencies.

rs ID: http://www.ncbi.nlm.nih.gov/snp/.

'+' symbol indicates the wild-type allele; 'del' denotes the allele characterized by the deletion.

FN3K, fructosamine-3-kinase; rs, RefSNP; T2DM, type 2 diabetes mellitus.

(clinical records, ECG, and echocardiograms) and classified as absent or present.

Sample size estimation of this exploratory study was based on an empirical assessment due to lack of knowledge of the frequency or values of parameters investigated.

\section{Molecular analysis}

DNA was extracted from subjects' whole blood samples using the QIAamp DNABlood Mini Kit (QIAGEN, Hilden, Germany). The procedure consists of four steps and was automated using the QIAcube (QIAGEN), a tool capable of processing QIAGEN balusters that enables 


\begin{tabular}{|c|c|c|c|c|}
\hline Genotypes & $\begin{array}{l}\text { c. }-385 A>G \\
\text { (rs3859206) }\end{array}$ & $\begin{array}{l}\text { c. }-232 A>T \\
\text { (rs2256339) }\end{array}$ & $\begin{array}{l}\text { c. } 900 C>G \\
\text { (rs1056534) }\end{array}$ & $\begin{array}{l}\text { T2DM } \\
(\mathrm{n}=80)\end{array}$ \\
\hline$A$ & GG & $T T$ & $\mathrm{CC}$ & 0.1000 \\
\hline B & GA & TA & $C G$ & 0.2875 \\
\hline C & $A A$ & $A A$ & $G G$ & 0.1375 \\
\hline D & $A A$ & $\mathrm{TA}$ & $C G$ & 0.0375 \\
\hline$E$ & $A A$ & $\mathrm{TA}$ & GG & 0.1000 \\
\hline $\mathrm{F}$ & $G G$ & $\mathrm{TA}$ & $C G$ & 0.0500 \\
\hline G & GA & $A A$ & GG & 0.0750 \\
\hline $\mathrm{H}$ & GA & TA & GG & 0.0500 \\
\hline I & GA & TA & $\mathrm{CC}$ & 0.0500 \\
\hline J & $A A$ & AA & $C G$ & 0.0250 \\
\hline $\mathrm{K}$ & $A A$ & $\mathrm{TT}$ & GG & 0.0125 \\
\hline L & GA & $T T$ & $C G$ & 0.0375 \\
\hline M & GA & $A A$ & CG & 0.0375 \\
\hline
\end{tabular}

Values for genotypes are frequencies. Frequencies $\geq 0.1$ are in italics. rs ID: http://www.ncbi.nlm.nih.gov/snp/.

rs, RefSNP; T2DM, type 2 diabetes mellitus.

automated low-yield sample preparation. Each whole blood sample was thawed and $200 \mu \mathrm{L}$ was used for DNA extraction to obtain 3-12 $\mathrm{g}$ g of DNA, which was then amplified by PCR. Primers were designed to amplify the promoter region and the six exons, and corresponding intron/exon boundaries of the $F N 3 K$ gene $^{20}$ in order to avoid amplifying any pseudogene located on chromosome 22. ${ }^{21}$ Protocols specifically developed at our laboratory were used to amplify the largest number of exons with the same protocol. Because of its length, exon 6 was divided into two parts and amplified with two different PCR reactions. Online supplementary table 1 shows the length of the PCR products and some characteristics of the primers (sequence and melting temperature) used in each PCR amplification. The amplification protocol was completed using Biomek NXP (Beckman Coulter). The PCR products were then purified using the AMPure PCR Purification Kit (Agencourt Bioscience Corporation), automating the process with the Biomek 3000 (Beckman Coulter). Purified amplicons of the $F N 3 K$ gene were then sequenced on both strands. The reaction plate with the sequencing mix was again automated using the Biomek 3000 (Beckman Coulter). Sequencing reaction products were purified using CleanSEQ Sequencing Reaction Clean-Up through the Biomek 3000 (Beckman Coulter). Finally, samples containing the sequencing reactions were loaded on a 48-capillary 3730 DNA Analyzer (Applied Biosystem), and the raw data were analyzed with the Sequencing Analysis software.

\section{Statistical analysis}

Data were expressed as mean \pm SD. The statistical analysis was performed using JMP13 software for Windows (SAS Institute, Cary, NC, USA). Student's $t$-test for independent samples was used to compare the data relating to the anthropometric and biohumoral parameters, as well as risk markers. The total complications were analyzed with the non-parametric Kruskal-Wallis test, followed by post hoc analysis. The $\chi^{2}$ test was used to compare qualitative data. A $p$ value of $<0.05$ was considered statistically significant.

The allelic frequencies for each polymorphism were calculated. The Heardy-Weinberg equilibrium (HWE) of the polymorphisms identified was estimated using the $\chi^{2}$ test. The statistical analysis of our sample indicated that the HWE was respected for all the polymorphisms detected.

\section{RESULTS}

Table 1 shows the anthropometric, clinical and biochemical characteristics of the 80 subjects with T2DM enrolled in the present study, and the overall distribution of their microangiopathic and macroangiopathic complications.

All subjects were tested for the $F N 3 K$ gene, considering all six exons with the corresponding intron-exon boundaries, and the promoter region. This screening identified 13 variants within the $F N 3 K$ gene, 3 of which (c.2T >A, IVS2-27A $>$ G and c.465G $>$ A) were never previously reported. In addition, one of our subjects with T2DM revealed two variants previously identified in a single patient with type 1 diabetes mellitus (c.-421C $>$ T and c.-429delATCGGAG) ${ }^{22}$ (see table 2).

Referring to the literature, we sought genotypes comprising polymorphisms most associated with a variation in the enzymatic activity of FN3K in erythrocytes ${ }^{23}$ in combination with a variation in HbAlc values. ${ }^{13}$ The polymorphisms concerned are rs3859206, rs2256339 (in the promoter region), and rs1056534 (in exon 6) (table 2). The thirteen genotypes identified, labeled A to $\mathrm{M}$, are listed in table 3. The genotypes GG at position -385 , TT at position -232 , and CC at c.900 have been associated with a better performance of the FN3K enzymatic activity. ${ }^{23}$ The last one has been also associated with lower HbAlc levels. ${ }^{13}$

Only four of the genotypes (A, B, C, and E) had a frequency $\geq 0.1$, so the others were grouped together. The clinical data collected for the study (population, anthropometric and hematochemical parameters, markers of risk, and microangiopathic and macroangiopathic complications) were associated with the five resulting genotype groups (A, B, C, E and Others) (table 4). The comparison between genotype groups revealed no statistically significant differences for any of the parameters considered, apart from age, glomerular filtration rate and vitamin E levels. In particular, genotype A was associated with a significantly older age than genotype $\mathrm{C}$ $(p=0.0050)$ or the Others $(p=0.0252)$, and genotype $\mathrm{C}$ was associated with a significantly younger age than genotype $\mathrm{E}(p=0.0445)$. Moreover, genotype A was associated with significantly higher vitamin $\mathrm{E}$ levels than genotype B $(p=0.0365)$. 
Table 4 Subjects' clinical characteristics by FN3K genotype group; for continuous data, values are mean \pm SD

\begin{tabular}{|c|c|c|c|c|c|}
\hline Parameter & A genotype & B genotype & C genotype & E genotype & Others \\
\hline $\mathrm{n}$ & 8 & 23 & 11 & 8 & 30 \\
\hline Age (years) & $74 \pm 8^{\mathrm{C}, \text { Others }}$ & $69 \pm 8$ & $64 \pm 7^{\mathrm{E}}$ & $71 \pm 7$ & $68 \pm 7$ \\
\hline Gender, M/F & $4 / 4$ & $8 / 15$ & $7 / 4$ & $5 / 3$ & $17 / 13$ \\
\hline Diabetes duration (years) & $12.4 \pm 4.0$ & $14.6 \pm 4.5$ & $15.7 \pm 3.9$ & $13.1 \pm 4.6$ & $15.1 \pm 4.2$ \\
\hline $\mathrm{BMI}\left(\mathrm{kg} / \mathrm{m}^{2}\right)$ & $31 \pm 5$ & $30 \pm 6$ & $31 \pm 5$ & $29 \pm 4$ & $30 \pm 5$ \\
\hline Waist circumference $(\mathrm{cm})$ & $102 \pm 8$ & $103 \pm 12$ & $105 \pm 13$ & $100 \pm 7$ & $101 \pm 11$ \\
\hline Systolic blood pressure $(\mathrm{mm} \mathrm{Hg})$ & $135 \pm 12$ & $135 \pm 12$ & $138 \pm 13$ & $139 \pm 12$ & $137 \pm 10$ \\
\hline Diastolic blood pressure $(\mathrm{mm} \mathrm{Hg})$ & $79 \pm 6$ & $80 \pm 9$ & $79 \pm 7$ & $80 \pm 10$ & $81 \pm 7$ \\
\hline DCCT-HbA1c (\%) & $7.2 \pm 0.7$ & $7.6 \pm 1.0$ & $7.3 \pm 0.5$ & $7.7 \pm 1.1$ & $7.2 \pm 0.7$ \\
\hline IFCC-HbA1c $(\mathrm{mmol} / \mathrm{mol})$ & $54.8 \pm 7.6$ & $59.3 \pm 11.3$ & $56.0 \pm 6.2$ & $59.6 \pm 14.0$ & $54.9 \pm 6.9$ \\
\hline Total cholesterol (mg/dL) & $181 \pm 33$ & $180 \pm 48$ & $158 \pm 28$ & $188 \pm 23$ & $179 \pm 32$ \\
\hline HDL cholesterol (mg/dL) & $56 \pm 20$ & $57 \pm 18$ & $55 \pm 10$ & $47 \pm 9$ & $50 \pm 14$ \\
\hline Triglycerides (mg/dL) & $131 \pm 57$ & $105 \pm 47$ & $96 \pm 36$ & $131 \pm 41$ & $128 \pm 61$ \\
\hline Microalbuminuria, yes/no & $8 / 0$ & $3 / 20$ & $3 / 8$ & $3 / 5$ & $7 / 23$ \\
\hline Albuminuria $(\mathrm{mg} / \mathrm{g})$ & $5 \pm 4$ & $31 \pm 65$ & $22 \pm 21$ & $19 \pm 19$ & $28 \pm 34$ \\
\hline Creatininemia $(\mathrm{mg} / \mathrm{dL})$ & $0.9 \pm 0.3$ & $0.8 \pm 0.2$ & $0.9 \pm 0.2$ & $1.0 \pm 0.2$ & $0.8 \pm 0.2$ \\
\hline Glomerular filtration rate $(\mathrm{mL} / \mathrm{min})$ & $77 \pm 16$ & $84 \pm 20$ & $82 \pm 17$ & $71 \pm 14$ & $89 \pm 21^{E}$ \\
\hline Homocysteine $(\mu \mathrm{mol} / \mathrm{L})$ & $17 \pm 3$ & $16 \pm 5$ & $18 \pm 8$ & $18 \pm 7$ & $15 \pm 5$ \\
\hline Vitamin A ( $\mu \mathrm{g} / \mathrm{dL})$ & $56 \pm 15$ & $51 \pm 15$ & $45 \pm 10$ & $52 \pm 10$ & $52 \pm 17$ \\
\hline Vitamin E (mg/dL) & $1.5 \pm 0.3^{B}$ & $1.3 \pm 0.3$ & $1.3 \pm 0.3$ & $1.3 \pm 0.2$ & $1.3 \pm 0.3$ \\
\hline Cerebral macroangiopathy, yes/no & $4 / 4$ & $16 / 7$ & $5 / 6$ & $5 / 3$ & $20 / 10$ \\
\hline Coronary heart disease, yes/no & $0 / 8$ & $3 / 20$ & $1 / 10$ & $0 / 8$ & $0 / 30$ \\
\hline Retinopathy, yes/no & $0 / 8$ & $5 / 18$ & $5 / 6$ & $1 / 7$ & $11 / 19$ \\
\hline Peripheral artery disease, yes/no & $0 / 8$ & $3 / 20$ & $1 / 10$ & $2 / 8$ & $5 / 25$ \\
\hline
\end{tabular}

Superscript letters indicate significant differences between respective genotype groups.

BMI, body mass index; DCCT-HbA1c, HbA1c according to Diabetes Control and Complications Trial units; F, female; FN3K, fructosamine-3-

kinase; HDL, high-density lipoprotein; IFCC-HbA1c, HbA1c according to International Federation of Clinical Chemistry units; M, male.

Microangiopathic and macroangiopathic complications were considered together, and their frequency was compared between the different genotype groups (figure 1). The non-parametric Kruskal-Wallis test indicated a significantly different number of complications among the groups $(p=0.0306)$. Following a post hoc analysis, a significant difference emerged between genotype A and genotypes $\mathrm{B}(p=0.0145), \mathrm{C}(p=0.0273), \mathrm{E}(p=0.0373)$, and the Others $(p=0.0024)$.

\section{DISCUSSION}

Recent years have seen a growing role of precision medicine in efforts to identify prevention, diagnosis and treatment strategies targeting particular categories of patients, stratifying populations by applying genomics to develop individual phenotypic profiles of disease. ${ }^{24}$ In the field of diabetes, one of the currently most intriguing fields of investigation focuses on glycation. Several studies have shown that glycation is associated with a condition of chronic hyperglycemia and the consequent development of long-term complications of diabetes. ${ }^{25}$ In this setting, the identification of FN3K (an enzyme capable of preventing the effects of hyperglycemia by intervening on protein glycation, and thereby on the damage mechanisms responsible for the onset of diabetic complications) has aroused great interest. ${ }^{26}$

In this exploratory study, the $F N 3 K$ gene was analyzed by direct sequencing in a cohort of 80 patients with T2DM. Three polymorphisms within the FN3K gene, found relevant in the literature, ${ }^{1322} 2327$ were taken into consideration: c.-385A $>\mathrm{G}$ (rs3859206) and c.-232A $>\mathrm{T}$ (rs2256339), located in the promoter region, and c.900C $>\mathrm{G}$ (rs1056534), located on exon 6 (table 2). Thirteen genotypes were identified (table 3 ), and clinical data were compared by genotype (table 4 ).

No significant differences in subjects' demographic, anthropometric or biohumoral parameters emerged between the different genotype groups (with the exceptions of older age and higher vitamin $\mathrm{E}$ levels for the genotype $\mathrm{A}$, and glomerular filtration rate between genotype $\mathrm{E}$ and Others). A similar trend was seen for the risk factors investigated. 


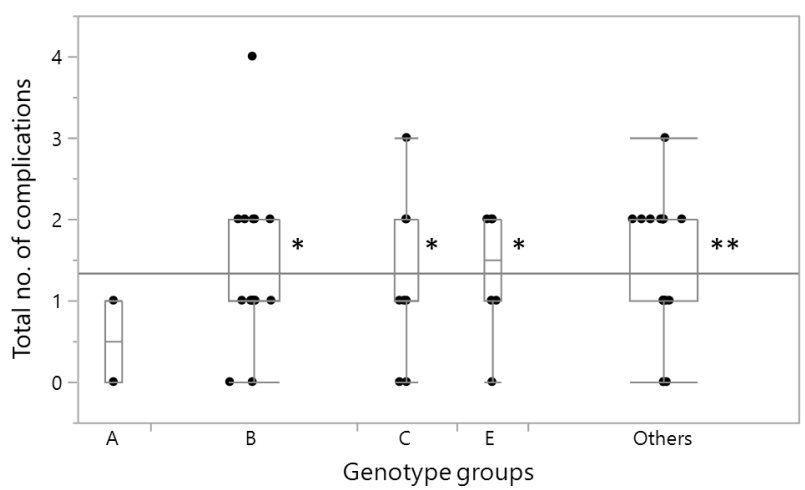

Figure 1 Box plot showing the distribution of the combined microangiopathic and macroangiopathic complications in the FN3K genotype groups. The total number of complications per patient includes the presence of at least one of the following: cerebral macroangiopathy, peripheral artery disease, ischemic heart disease, retinopathy, and microalbuminuria. The Kruskal-Wallis test indicated a statistically significant difference among the genotype groups $(p=0.0306) .{ }^{*} p<0.01,{ }^{*} p<0.05$ post hoc analysis versus genotype group $A$. In the graph, the edges of the box indicate the 25th and 75th quantiles, including the middle $50 \%$ of the data; whiskers indicate the range of data, calculated as [upper quartile+1.5 (IQR)] and [lower quartile-1.5 (IQR)]; the continuous horizontal line is the overall arithmetical mean for all the data; the scattered points are single subject's values. The width of each box is proportional to the number of cases in each group. FN3K, fructosamine-3-kinase.

Intriguingly, when microangiopathic and macroangiopathic complications were pooled together, genotype A (deriving from the combination of the favorable alleles GG in c. $-385 \mathrm{~A}>\mathrm{G}$, TT in c.-232A $>\mathrm{T}$, and $\mathrm{CG}$ in c.900C $>\mathrm{G}$ ) showed a statistically significant inverse relationship with their occurrence. This might mean that genotype A could be a factor in preventing the onset of vascular complications. To the best of our knowledge, this finding is new and in line with previous studies on FN3K. In particular, Delpierre $e t a l^{23}$ reported that, when analyzed individually, the alleles in genotype A were associated with a better performance of the enzymatic activity of FN3K, coinciding with a lower level of glycation. Škrha et $a l^{15}$ correlated the GG allele of the c.900 $\mathrm{C}>\mathrm{G}$ polymorphism with a greater production of soluble receptors for advanced glycation end products. Our study suggests that the CC allele also contributes to the protective effect associated with genotype A.

The severity of long-term diabetic complications is usually age-related. ${ }^{28}$ Intriguingly, patients with genotype A and with a better outcome in terms of vascular complications are older than the patients belonging to the other genotypic groups. This observation indirectly reinforces our finding of a protective role of FN3K in the development of vascular complications of diabetes.

One of the limitations of our study concerns the small sample size of the genotype groups, which may explain the lack of significant differences between the various genotypes when microvascular and macrovascular complications were analyzed separately. However, the subdivision into genotype groups was not predictable a priori, and therefore the reduced number of subjects resulting from the initial T2DM cohort unfortunately caused a reduction of the study power. Despite this limitation, there was an evident difference in overall microangiopathic and macroangiopathic complications between genotype A and all the other genotypes. The genotype effect on individual complications, leading to nonsignificant results for the complications considered separately, would deserve further investigation, also aimed at evaluating the potentially protective effect of genotype A on FN3K activity in a larger cohort. In this light, the recent report from Dunmore et $a l^{29}$ is highly relevant, as it demonstrates a relationship between the enzymatic activity of FN3K and the glycation gap. Compared with HbAlc levels, the glycation gap is a more reliable indicator of glyco-oxidative stress (and the consequent risk of developing complications), linked to predictions based on fructosamine levels. ${ }^{830}{ }^{31}$ It will be worth integrating the $F N 3 K$ genetic variants with the glycation gap to shed more light on the possible protective role of genotype A that emerged from our study.

Another limitation of our study regarding the possible determination of the deglycating products of the FN3K variants we have genotyped. Indeed, we performed some experiments to measure the FN3K catalytic activity by adopting the procedure described by Krause $e t a l^{32}$ based on the conversion of the synthetic UV-active fructosamine No-hippuryl-Ne-(1-deoxy-D-fructosyl)lysine (BzGFruK) to No-hippuryl-Ne-(phosphofructosyl) lysine (BzGpFruK). The substrate has been synthetized, but the phosphorylated substrate was very unstable and very difficult to be quantified in a reproducible way. A more promising and simple method was developed later by another group,$^{33}$ but we were not convinced of the substrate these authors were using (ie, bovine albumin glycated in vitro) because previous experience with this substrate also showed its marked instability. So, at least according to our experience, the determination of FN3K remains still an open issue.

The identification of a genotype with a protective role may open up new prospects for research on $F N 3 K$ genetic variability and on its potential applicability to the prevention, diagnosis and treatment of diabetes and its complications.

\section{Author affiliations}

${ }^{1}$ Department of Medicine (DIMED), University of Padova School of Medicine and Surgery, Padova, Italy

${ }^{2}$ Department of Pharmaceutical and Pharmacological Sciences, University of Padova School of Medicine and Surgery, Padova, Italy

${ }^{3}$ Department of Pathophysiology and Transplantation, University of Milan, Milano, Italy

${ }^{4}$ Istituto di Tecnologie Biomediche, Consiglio Nazionale delle Ricerche (ITB-CNR), Milan, Italy

Contributors GS designed the study, interpreted the data and revised the manuscript. AM, RP and FA carried analyses and contributed to interpretation of data. ER analyzed and interpreted the data, and wrote the manuscript. NCC and 
SB provided patient samples and contributed to the discussion. AL reviewed and critically edited the manuscript.

Funding This work was supported by Department of Medicine, University of Padua, and by Ministero dell'Istruzione, dell'Università e della Ricerca.

Competing interests None declared.

Patient consent for publication Not required

Ethics approval The study used information that is available in the database of the Department of Medicine (DIMED), University of Padua. The study was approved by ethical committee for clinical trials in Padua (approval no.149 DCCT/HBA1C) and was conducted in accordance with the Helsinki Declaration. Participants gave informed consent before taking part to the study.

Provenance and peer review Not commissioned; externally peer reviewed.

Data availability statement All data relevant to the study are included in the article or uploaded as supplementary information.

Open access This is an open access article distributed in accordance with the Creative Commons Attribution Non Commercial (CC BY-NC 4.0) license, which permits others to distribute, remix, adapt, build upon this work non-commercially, and license their derivative works on different terms, provided the original work is properly cited, appropriate credit is given, any changes made indicated, and the use is non-commercial. See: http://creativecommons.org/licenses/by-nc/4.0/.

ORCID iD

Eugenio Ragazzi http://orcid.org/0000-0002-0390-6823

\section{REFERENCES}

1 Zhou B, Lu Y, Hajifathalian K, et al. Worldwide trends in diabetes since 1980: a pooled analysis of 751 population-based studies with 4.4 million participants. Lancet 2016;387:1513-30.

2 Saeedi P, Petersohn I, Salpea P, et al. Global and regional diabetes prevalence estimates for 2019 and projections for 2030 and 2045 : Results from the International Diabetes Federation Diabetes Atlas, $9^{\text {th }}$ edition. Diabetes Res Clin Pract 2019;157:107843

3 Stratton IM, Adler Al, Neil HA, et al. Association of glycaemia with macrovascular and microvascular complications of type 2 diabetes (UKPDS 35): prospective observational study. BMJ 2000;321:405-12.

4 Brownlee M. Biochemistry and molecular cell biology of diabetic complications. Nature 2001;414:813-20.

5 Sheetz MJ, King GL. Molecular understanding of hyperglycemia's adverse effects for diabetic complications. JAMA 2002;288:2579-88.

6 Stitt AW, Jenkins AJ, Cooper ME. Advanced glycation end products and diabetic complications. Expert Opin Investig Drugs 2002;11:1205-23.

7 World Health Organization. Use of glycated haemoglobin (HbA1c) in the diagnosis of diabetes mellitus [Internet], 2011. Available: http:// www.who.int/diabetes/publications/diagnosis_diabetes2011/en/ [Accessed 15 Jan 2020].

8 Cohen RM, Holmes YR, Chenier TC, et al. Discordance between $\mathrm{HbA1c}$ and fructosamine: evidence for a glycosylation gap and its relation to diabetic nephropathy. Diabetes Care 2003;26:163-7.

9 Ames JM. The Maillard reaction. In: Hudson BJF, ed. Biochemistry of food proteins. London: Elsevier Appl. Sci, 1992: 99-153.

10 Zhang Q, Ames JM, Smith RD, et al. A perspective on the Maillard reaction and the analysis of protein glycation by mass spectrometry: probing the pathogenesis of chronic disease. J Proteome Res 2009;8:754-69.

11 Szwergold BS, Howell S, Beisswenger PJ. Human fructosamine-3kinase: purification, sequencing, substrate specificity, and evidence of activity in vivo. Diabetes 2001;50:2139-47.

12 Van Schaftingen E, Collard F, Wiame E, et al. Enzymatic repair of Amadori products. Amino Acids 2012;42:1143-50.

13 Mohás $M$, Kisfali $P$, Baricza $E$, et al. A polymorphism within the fructosamine-3-kinase gene is associated with $\mathrm{HbA1c}$ levels and the onset of type 2 diabetes mellitus. Exp Clin Endocrinol Diabetes 2010;118:209-12.

14 Veiga da-Cunha M, Jacquemin P, Delpierre G, et al. Increased protein glycation in fructosamine 3-kinase-deficient mice. Biochem J 2006;399:257-64.

15 Škrha J, Muravská A, Flekač M, et al. Fructosamine 3-kinase and glyoxalase I polymorphisms and their association with soluble RAGE and adhesion molecules in diabetes. Physiol Res 2014;63:S283-91.

16 World Health Organization \& International Diabetes Federation. Definition and diagnosis of diabetes mellitus and intermediate hyperglycaemia: report of a WHO/IDF consultation. Geneva: World Health Organization, 2006. http://www.who.int/iris/handle/10665/ 43588

17 American Diabetes Association. 2. Classification and Diagnosis of Diabetes: Standards of Medical Care in Diabetes-2019. Diabetes Care 2019;42:S13-28.

18 Danaei G, Finucane MM, Lu Y, et al. National, regional, and global trends in fasting plasma glucose and diabetes prevalence since 1980: systematic analysis of health examination surveys and epidemiological studies with 370 country-years and 2.7 million participants. Lancet 2011;378:31-40.

19 Piarulli F, Sartore G, Ceriello A, et al. Relationship between glycooxidation, antioxidant status and microalbuminuria in type 2 diabetic patients. Diabetologia 2009;52:1419-25.

20 Alexander RP, Fang G, Rozowsky J, et al. Annotating non-coding regions of the genome. Nat Rev Genet 2010;11:559-71.

21 Collard F, Delpierre G, Stroobant V, et al. A mammalian protein homologous to fructosamine-3-kinase is a ketosamine-3-kinase acting on psicosamines and ribulosamines but not on fructosamines. Diabetes 2003;52:2888-95.

22 Avemaria F, Carrera P, Lapolla A, et al. Possible role of fructosamine 3-kinase genotyping for the management of diabetic patients. Clin Chem Lab Med 2015;53:1315-20.

23 Delpierre G, Veiga-da-Cunha M, Vertommen D, et al. Variability in erythrocyte fructosamine 3-kinase activity in humans correlates with polymorphisms in the FN3K gene and impacts on haemoglobin glycation at specific sites. Diabetes Metab 2006;32:31-9.

24 Klonoff DC. Precision medicine for managing diabetes. J Diabetes Sci Technol 2015;9:3-7.

25 Brings S, Fleming T, Freichel M, et al. Dicarbonyls and advanced glycation end-products in the development of diabetic complications and targets for intervention. Int J Mol Sci 2017;18. doi:10.3390/ ijms18050984. [Epub ahead of print: 05 May 2017].

26 Conner JR, Beisswenger PJ, Szwergold BS. The expression of the genes for fructosamine-3-kinase and fructosamine-3kinase-related protein appears to be constitutive and unaffected by environmental signals. Biochem Biophys Res Commun 2004;323:932-6.

27 Mosca L, Penco S, Patrosso MC, et al. Genetic variability of the fructosamine 3-kinase gene in diabetic patients. Clin Chem Lab Med 2011;49:803-8.

28 Shamshirgaran SM, Mamaghanian A, Aliasgarzadeh A, et al. Age differences in diabetes-related complications and glycemic control. BMC Endocr Disord 2017;17:25.

29 Dunmore SJ, Al-Derawi AS, Nayak AU, et al. Evidence that differences in fructosamine-3-kinase activity may be associated with the glycation gap in human diabetes. Diabetes 2018;67:131-6.

30 Macdonald DR, Hanson AM, Holland MR, et al. Clinical impact of variability in $\mathrm{HbA} 1 \mathrm{c}$ as assessed by simultaneously measuring fructosamine and use of error grid analysis. Ann Clin Biochem 2008;45:421-5.

31 Nayak AU, Holland MR, Macdonald DR, et al. Evidence for consistency of the glycation gap in diabetes. Diabetes Care 2011;34:1712-6.

32 Krause R, Oehme A, Wolf K, et al. A convenient HPLC assay for the determination of fructosamine-3-kinase activity in erythrocytes. Anal Bioanal Chem 2006;386:2019-25.

33 Cikomola JC, Kishabongo AS, Vandepoele K, et al. A simple colorimetric assay for measuring fructosamine 3 kinase activity. Clin Chem Lab Med 2017;55:154-9. 